\title{
Spectroscopy of ultrathin epitaxial rutile TiO[sub 2](110) films grown on W(100)
}

Article

Accepted Version

Bennett, R. A., Mulley, J., Newton, M.A. and Surman, M. (2007) Spectroscopy of ultrathin epitaxial rutile TiO[sub 2] (110) films grown on $\mathrm{W}(100)$. The Journal of Chemical Physics, 127 (8). 084707. ISSN 0021-9606 doi: https://doi.org/10.1063/1.2756842 Available at https://centaur.reading.ac.uk/16788/

It is advisable to refer to the publisher's version if you intend to cite from the work. See Guidance on citing.

To link to this article DOI: http://dx.doi.org/10.1063/1.2756842

Publisher: American Institute of Physics

All outputs in CentAUR are protected by Intellectual Property Rights law, including copyright law. Copyright and IPR is retained by the creators or other copyright holders. Terms and conditions for use of this material are defined in the End User Agreement.

\section{www.reading.ac.uk/centaur}

\section{CentAUR}

Central Archive at the University of Reading 
Reading's research outputs online 


\title{
Spectroscopy of ultra-thin epitaxial rutile $\mathrm{TiO}_{2}(110)$ films grown on $\mathrm{W}(100)$
}

\author{
R.A. Bennett, J.S. Mulley \\ Department of Physics, University of Reading, Whiteknights, Reading, RG6 6AF, United Kingdom
}

M.A. Newton

The European Synchrotron Radiation Source, 6 Rue Jules Horowitz, B.P. 220, 38043 Grenoble,

France

M. Surman

CCLRC Synchrotron Radiation Source, Daresbury, Cheshire, WA4 2AD, United Kingdom

\begin{abstract}
Epitaxial ultra-thin titanium dioxide films of 0.3 to $\sim 7 \mathrm{~nm}$ thickness on a metal single crystal substrate have been investigated by high resolution vibrational and electron spectroscopies. The data complement previous morphological data provided by scanned probe microscopy and low energy electron diffraction to provide very complete characterisation of this system. The thicker films display electronic structure consistent with a stoichiometric $\mathrm{TiO}_{2}$ phase. The thinner films appear nonstoichiometric due to band bending and charge transfer from the metal substrate while workfunction measurements also show a marked thickness dependence. The vibrational spectroscopy shows three clear phonon bands at $368 \mathrm{~cm}^{-1}, 438 \mathrm{~cm}^{-1}$ and $829 \mathrm{~cm}^{-1}$ (at $273 \mathrm{~K}$ ) which confirms a rutile structure. The phonon bands intensity scales linearly with film thickness and shift slightly to lower frequencies with increasing temperature, in accord with results for single crystals.
\end{abstract}




\section{Introduction}

Transition metal oxide surfaces have recently become an area of intense interest due to their fundamental importance in applications such as heterogeneous catalysis, as dielectrics and magnetic tunnel junctions in solid state electronic devices, on structural metal ceramic interfaces and as gas sensors for example. Titanium dioxide in particular has become the prototypical system for study due to its relatively simple electronic structure and accessibility in single crystalline form [1]. However, the many disparate roles the material plays are sensitive to the nature of its free surfaces and oxide-metal interfaces. In particular non-stoichiometry tends to lead to a semi-conducting bulk and novel surface chemistry. Strong departures from stoichiometry can also lead to ordered crystallographic shear plane phases in the bulk [2] which give ordered surface structures [3-7]. Reproducible preparation and interrogation of stoichiometric $\mathrm{TiO}_{2}(110)$ surfaces is difficult as non-stoichiometry is required to make the crystal sufficiently conducting for surface electron spectroscopy and scanning tunnelling microscopy. Similar problems arise with investigations of wide band gap oxides such as $\mathrm{Al}_{2} \mathrm{O}_{3}, \mathrm{MgO}$ and $\mathrm{SiO}_{2}$ and here epitaxial layers on metals have proved to be very effective in providing sufficiently high electron tunnelling probabilities to prevent film charging. However, for oxides with the rutile structure the high aspect ratio of the principal lattice vectors (2.19) of the most stable face (110) precludes simple epitaxial match to most metals.

The properties of ultra-thin films may not necessarily follow their bulk counterparts, for example $\mathrm{MgO}$ has differing adsorption behaviour for water and metal adatoms to that the bulk truncated surface $[8,9]$ and thin film silicon oxides can form crystalline lattices whereas the bulk and native oxides are amorphous glassy materials [10]. We have recently reported on a methodology for the formation of 
epitaxial rutile $\mathrm{TiO}_{2}$ in the (110) orientation on the $\mathrm{W}(100)$ surface which allows the creation of well defined films for further study [11]. The control afforded by our methodology can be used to produce stoichiometric phases of other rutile reducible oxides in a controlled manner, most notably $\mathrm{CrO}_{2}[12]$.

$\mathrm{TiO}_{2}$-metal interfaces have received some attention due to the metal-support interactions resulting in novel surface chemistries. However in this case the metal is a nanoparticulate phase supported on a bulk oxide material. $\mathrm{TiO}_{\mathrm{x}}$ ultra-thin films have been reported on a number of surfaces although their composition and homogeneity are rarely confirmed in detail [13-16]. Theoretical studies of these systems is a very active area with oxide thin films predicted to have novel properties and stability, especially at the Nanoscale [17]. For example through slight expansion $(0.8 \%)$ of the $a=b$ lattice constant $\mathrm{TiO}_{2}$ is predicted [18] to become strongly ferroelectric with a tenfold increase in c-axis dielectric constant. The authors suggest such an expansion of the lattice could be obtained by epitaxial growth with small lattice mismatch. First principles calculations for oxide films predict workfunction changes at the metal through a variety of mechanisms with the $\mathrm{TiO}_{2} / \mathrm{Mo}(100)$ surface involving charge transfer from metal to film and a concomitant increase in workfuction [19].

In this paper we detail the growth of electronic and vibrational properties of ultra-thin film $\mathrm{TiO}_{2}(110)$ grown on $\mathrm{W}(100)$. The experimental data extends our previous study which concentrated on detailing the growth characterising the geometrical structure of thicker ultra-thin films $(>2 \mathrm{~nm})[11,20]$. That work showed by LEED (and some ex-situ STM) that the films grew epitaxially aligned in the (110) orientation, covered the surface and had step heights of $3.2 \AA$ as per rutile $\mathrm{TiO}_{2}$, making this system one of the best characterised $\mathrm{TiO}_{\mathrm{x}}$ films. By investigation of near monolayer films and detailed UPS measurements here we can discern finer points that 
distinguish the properties of the films from bulk behaviour and provide key data to support theoretical efforts.

\section{Experimental}

The experiments were carried out in two separate UHV systems and were equipped with same LEED system, Oxford Applied Research water-cooled e-beam evaporator Ti source and sample mounting. Electron spectroscopy was undertaken in a twin chamber UHV system, described previously [11] but recently upgraded through improved turbo and ion pumping. One chamber is equipped for sample preparation with a Vacuum Generators 3 grid retarding field analyser for low energy electron diffraction (LEED), a quadrupole mass spectrometer, the Ti source and additional facilities for $\mathrm{Ar}^{+}$or $\mathrm{O}^{+}$ion sputtering. The second chamber is mu metal and equipped with a hemispherical electron energy analyser, which can be used in conjunction with an $\mathrm{Al} \mathrm{K \alpha} \mathrm{x}$-ray source to perform x-ray photoelectron spectroscopy (XPS), or with a Helium lamp to perform ultra-violet photoelectron spectroscopy (UPS). A gate valve separates the two chambers so that the analyser is never exposed to large reactive gas loads that might change its workfunction.

The second UHV system is mounted on Beamline 13.3 of the Daresbury synchrotron radiation facility and has been described in detail [21, 22]. A close-in mirror collects $60 \times 60 \mathrm{mrad}$ of light from the $2 \mathrm{GeV}$ electron storage ring. This radiation is focussed at a wedged diamond window which isolates the storage ring vacuum. The light is then collimated into the custom Thermo-Nicolet interferometer maintained at $5 \times 10^{-2}$ mbar to eliminate water vapour. The light is then focussed through CsI windows into the centre of the UHV surface science chamber (base 
pressure $2 \times 10^{-10} \mathrm{mBar}$ ) where it is reflected at grazing incidence from the sample. The diverging beam emerges through a second CsI window into rough vacuum and is refocused onto an Infrared Labs liquid helium cooled silicon bolometer. Fourier transform Infra Red (FT-IR) spectra were acquired at $2 \mathrm{~cm}^{-1}$ resolution during growth of the film and from the clean surface prior and post growth. The spectra were reprocessed such that the background spectra was the clean surface spectrum obtained immediately after the sample had regained the required temperature having been flashed in-situ to $2300 \mathrm{~K}$ to remove the film. This allowed for the lengthy growth, oxidation and movement of the sample for LEED analysis to not impact the spectroscopy of the film. Minute movements of the sample, upon heating or cooling for example, produced mis-cancellation of interference fringes in the detection optical system. This was eliminated by using a notch in the triangular apodisation function to remove the associated localised side-band in the sample and background interferograms. Some residual ripple does remain in spectra which is the limiting factor in the sensitivity.

The W(100) crystal is mounted and heated exactly as described previously [11] with temperature measurement by C-type thermocouple spot welded to the crystal. The W crystal was cleaned by repeated cycles of heating to $\sim 2300 \mathrm{~K}$, then annealing at $1300 \mathrm{~K}$ in $1 \times 10^{-7}$ Torr $\mathrm{O}_{2}$ for extended periods, then two flashes to $\sim 2300 \mathrm{~K}$ to remove residual oxygen. This was carried out until both the LEED pattern indicated a well-ordered $(1 \times 1)$ surface and the XPS showed the surface to be spectroscopically clean. The Ti was deposited at $\sim 2 / 3 \mathrm{ML}$ per minute onto the $\mathrm{W}$ surface maintained at $760 \mathrm{~K}$. The flux was monitored by the integral ion current meter in the source and periodically checked via monolayer breakpoint analysis by XPS. Oxidation of the films was achieved by exposing them to $1 \times 10^{-7}$ torr of high purity 
oxygen leaked into the chamber background by fine leak valve during deposition of the second and subsequent monolayers and also through post deposition annealing at the same temperatures and pressures. The gas purity was monitored by mass spectrometer. Film thickness' are referenced to the pseudomorphic Ti monolayer which is to within a small fraction the same number density as Ti in the stoichiometric rutile (110) surface.

\section{Results}

\section{Photoelectron spectroscopy}

\section{Stoichiometry}

Figure 1 contrasts XPS spectra of the Ti $2 p$ region for a $1 \mathrm{ML}$ and a $30 \mathrm{MLTiO}_{2}$ film grown on the W(100) substrate with 4 components fitted to each corresponding to the $\mathrm{Ti}^{4+}$ and $\mathrm{Ti}^{3+}$ ions for both of the spin orbit splits. The notionally $3^{+}$states are chemically core level shifted from the fully oxidised $\mathrm{Ti}^{4+}$. The components were fit to Gaussian Lorentzian product curves on a Shirley background using CASA XPS peak fitting software [23]. Table 1 shows the fitting parameters used systematically in this study to maintain reproducibility between analysis. The fraction of the $\mathrm{Ti}^{4+}$ peak area to the area of all the $\mathrm{Ti} 2 \mathrm{p}$ peaks gives the films stoichiometry.

The departure from $100 \%$ stoichiometry is due to the contribution of ions in apparent $\mathrm{Ti}^{3+}$ states which may be attributed to $\mathrm{W} / \mathrm{TiO}_{2}$ interface states, to defects arising from surface oxygen vacancies or from enrichment of Ti from interstitials. Stoichiometries determined for the range of film thicknesses deposited in this study are displayed in Figure 2. The shallow exponential decay curve fit to the $\mathrm{Ti}^{3+}$ with a value of $5.68 \pm 1.57 \mathrm{ML}$ for the mean free path on a constant $2.4 \pm 0.95 \%$ nonstoichiometric background. This agrees well with previous studies, which returned a 
value of 7ML [11], and in comparison to calculated values of 8ML [24]. For ultra-thin films $(<10 \mathrm{ML})$ a significant $\mathrm{Ti}^{3+}$ signal $(11 \pm 8 \%)$ arises localised at the interface, as we have previously discussed, and much of this paper is aimed at describing this in greater detail. The data above 10ML tend toward the upper limit of experimentally achievable stoichiometry ( $\sim 98 \%$ is generally the limit for bulk $\mathrm{TiO}_{2}$ prepared in $\mathrm{UHV}$ to give reasonable LEED patterns). For greater than $10 \mathrm{ML}$ films, where the $\mathrm{Ti}^{3+}$ filmsubstrate interface states are attenuated by the photoelectron escape depth, the residual $\mathrm{Ti}^{3+}$ states are attributed to surface and near surface point defects such as oxygen vacancies and Ti interstitials.

\section{UPS Spectra}

Ultra-violet photoelectron spectroscopy provides useful information of the valence band electronic states of transition metal oxides, especially formally $\mathrm{d}^{0}$ oxides where defects and dopants introduce $3 \mathrm{~d}$ states just below the Fermi level. In single crystal $\mathrm{TiO}_{2}$ the identification of the Fermi level is difficult as it is in the band gap and no emission is expected. One advantage of the thin film work is that the $\mathrm{W}$ substrate has a clear Fermi edge for referencing the peak positions and to measure the energy resolution $(\sim 100 \mathrm{meV}$ for all spectra here). He I $(\mathrm{hv}=21.21 \mathrm{eV})$ UPS spectra were recorded for all $\mathrm{TiO}_{2}$ coverages and for each clean W(100) starting substrate prior to deposition in order to obtain accurate reference spectra. For the $1 \mathrm{ML} \mathrm{TiO}_{2}$ film, the starting substrate was a $\mathrm{O}-\mathrm{W}(2 \times 1)$ reconstruction formed by oxidising the $\mathrm{W}$ crystal in $1 \times 10^{-7}$ torr $\mathrm{O}_{2}$ for $5 \mathrm{~min}$ followed by vacuum annealing at $1600^{\circ} \mathrm{C}$ for $10 \mathrm{~min}$. Figure 3 displays the three UPS typical spectra taken from the $1 \mathrm{ML} \mathrm{TiO}_{2}$ deposition.

The spectra are aligned at $0 \mathrm{eV}$ by the Fermi edge which is clear step function for the clean $\mathrm{W}(100)$ and remains visible in subsequent oxidation and growth. The 
tungsten surface is characterised by several surface states at $\sim 0.4,1.0,1.8$ and $4.5 \mathrm{eV}$ [25] (see figure 3). Some residual oxygen on the clean surface is also observed as a small peak $\sim 5 \mathrm{eV}$. The oxygen $2 \mathrm{p}$ states [26] of the $(2 \times 1)$ surface appear between 3 and $8 \mathrm{eV}$. Upon forming the monolayer Ti oxide film the onset of emission from the oxygen states that form the valence band is reduced from $3.15 \mathrm{eV}$ for the $\mathrm{O}-(2 \times 1)$ to $3.05 \mathrm{eV}$ for the $\mathrm{TiO}_{2} / \mathrm{W}$. The formation of $\mathrm{TiO}_{2}$ on the surface strengthens the intensity and broadens the emission from the oxygen valence states indicating an uptake of additional oxygen but a range of environments. The shape and width of the secondary electron tail and cutoff is also modified indicating a work function shift. However, there is negligable photoemission in the $0-3 \mathrm{eV}$ region of the $\mathrm{TiO}_{2}$ film. This is a surprising find since the XPS data indicate that these thin films are our least stoichiometric ( 91\%). One would expect to find Ti $3 \mathrm{~d}$ states $\sim 0.9 \mathrm{eV}$ binding energy for non-stoichiometric films, as found in non-stoichiometric single crystals and surfaces that have been modified by electron donating alkali metals etc $[27,28]$, Ti adatoms [29] and oxygen vacancies [30]. Furthermore the $\sim 3 \mathrm{eV}$ valence band offset, which is practically the entire bandgap of $3.05 \mathrm{eV}$, indicates that the Fermi level is pinned right at the bottom of the conduction band. These results would signify that the shift of the work function for the ultra thin films are interface derived and are not due to surface defects.

The development of structure in the $\mathrm{TiO}_{2}$ film spectra occurs with increasing film thickness. Figure 4 displays overlaid spectra for a $1 \mathrm{ML}$ and $30 \mathrm{ML} \mathrm{TiO}_{2}$ film normalised at the Fermi level. The thicker film also has distinct states apparent as observed for $\mathrm{TiO}_{2}(110)$ single crystal surfaces and can be assigned through first principles calculations to mainly antibonding bridging oxygen, mixed in plane oxygen and bulk oxygen orbitals and finally bonding orbitals from in plane and bulk oxygen 
with increasing binding energy [31]. The valence states of the 1ML film are less well defined, possibly as a result of lifetime broadening due to rapid quenching by the substrate of the valence hole left in the photoemission process. The onset of valence band emission shifts to lower binding energy with film thickness and converges on a value of $2.85 \pm 0.05 \mathrm{eV}$ at $\sim 5 \mathrm{ML}$. These offsets for the ultra-thin films with respect to the converged thick film values are displayed in Figure 2.

The work function, $\Phi$, of all films was calculated from the spectral width of the UPS spectra and the incident photon energy $(\mathrm{hv}=21.21 \mathrm{eV})$. The photoemission cut-off is found by measuring the intersection of the linear extrapolation of the secondary electron tail with a linear background fitted for the entire spectrum. The results are plotted in Figure 5 and show two distinct regimes. For films $<10 \mathrm{ML}$ we see a decrease in $\Phi$ from $\sim 6.3$ to $\sim 5.8 \mathrm{eV}$ with increasing thickness. For thicker films (>15ML) the value of $\Phi$ tends to a constant value of $\sim 5.8 \mathrm{eV}$. This limiting value agrees favourably with experimentally obtained values for the $\Phi$ of rutile $\mathrm{TiO}_{2}(110)$ which are variously quoted in the range $5.2-5.8 \mathrm{eV}$ [32-35] and is consistent with the notion of $>15 \mathrm{ML}$ films exhibiting pseudo-bulk properties. The range of values is due to the effect of surface defects with $\Phi$ decreasing with increasing defect density [36, 37]. Being at the upper end of the $\Phi$ range indicates that our prepared surfaces have a low defect density. This is further corroborated by measurements performed on a $\mathrm{TiO}_{2}(110)$ single crystal surface (which gave a $(1 \times 1)$ LEED pattern and was $98 \%$ stoichiometric as adjudged by XPS) where a value of $\Phi=5.75 \mathrm{eV}$ was found.

In order to understand the electronic structure of these thin films we highlight three important measurements: the onset of valence band emission with binding 
energy greater than or equal to the $3.05 \mathrm{eV}$ bandgap of $\mathrm{TiO}_{2}$ for films $<5 \mathrm{ML}$; the larger $\Phi$ for films $<5 \mathrm{ML}$ and the lack of $\mathrm{Ti} 3 \mathrm{~d}$ defect states at $0.9 \mathrm{eV}$ despite an apparent non-stoichiometry at the interface. These measurements can be explained by considering the band bending that occurs at the $\mathrm{W}-\mathrm{TiO}_{2}$ interface and by distinguishing the role of interface and surface effects that dominate the emission processes for ultra-thin $(<5 \mathrm{ML})$ and thin $(>5 \mathrm{ML})$ films.

The Fermi level is pinned at close to the bottom of the conduction band in all the films grown. The onset of photoemission from the valence band starts at $2.85 \mathrm{eV}$ below the Fermi level, which when combined with a $3.05 \mathrm{eV}$ bandgap implies the conduction band edge lies $0.2 \mathrm{eV}$ above the Fermi level. At the interface there is a downward bending of the bands as $\mathrm{W}(100)$ has a lower workfunction $(5 \mathrm{eV}$, measured by the same methodology as for the films) compared to the measured value of $5.85 \mathrm{eV}$ for $\mathrm{TiO}_{2}$ for the thickest films. In the Schottky limit with no external bias we find the built in interface potential, $V_{b i}$, to be equal to $-0.85 \mathrm{eV}$ (see Figure 6). Moreover, due to the pinning of the Fermi level at only $0.2 \mathrm{eV}$ beneath the conduction band minima, the oxide conduction band will actually cross the Fermi level at the interface. This causes a spill out of majority carriers (electrons) into the $\mathrm{TiO}_{2}$ film and creates an accumulation layer at the interface. These electrons are responsible for the apparent non-stoichiometry measured in the $\mathrm{Ti} 2 \mathrm{p}$ core states in the XPS experiments (the increased screening giving rise to the chemically core level shifted $\mathrm{Ti}^{3+}$ state) but are not apparent in the UPS data as the spill out electrons are in states originally unoccupied but now pinned at the Fermi-level of the metal. In effect the conduction band is bent until pinned by population of $\mathrm{Ti} 3 \mathrm{~d}$ states from the Fermi energy electrons of the metal - as soon as population is possible enough electrons are transferred to generate the interface potential at equilibrium. Thus the occupied $\mathrm{Ti} 3 \mathrm{~d}$ 
states straddle the Fermi edge and would be difficult to identify. New, defect states, that are significantly below the Fermi level are apparently not created at the interface.

We can, however, estimate the width of the depletion region, $W$, from the depletion approximation for a Schottky barrier [38]

$$
W=\sqrt{\frac{2 \varepsilon_{s} \varepsilon_{0} V_{b i}}{q N_{D}}}
$$

Where the relative permittivity of the $\mathrm{TiO}_{2}$ is $\varepsilon_{s}=11.9$ and the number density of acceptor sites is just the density of Ti in the oxide $N_{D}=3.2 \times 10^{28} \mathrm{~m}^{-3}$. This returns a value of $\sim 0.2 \mathrm{~nm}$ for the depletion width showing that it is likely to be confined to the first monolayer of the film. The filling of $\mathrm{Ti} 3 \mathrm{~d}$ states at the Fermi level by band bending at the interface therefore contrasts to UPS of defective and nonstoichiometric surfaces which have Ti $3 \mathrm{~d}$ derived defect states with binding energies of $0.9 \mathrm{eV}[29,39]$.

Charge transfer upon adsorption at surfaces leads to changes in the surface dipole moment and hence to workfunction changes. On $\mathrm{TiO}_{2}$ metal adsorption [36, 40] and charge transfer at ideal and defective surfaces is frequently implicated in changes of the workfunction and stoichiometry. Recent studies of workfunction changes and charge transfer in the N/W[100] system have shown that the electronic charge distribution of the entire surface region must be considered; the changes to the charge accumulation region and the height of the adsorbate must be compared to the overspill of electrons in $\mathrm{W} 5 \mathrm{~d}$ orbitals out of the clean surface [41]. The large increase in workfunction for the thinnest films here (from 5.0 to $6.33 \mathrm{eV}$ ) may therefore be due to the excess charge residing on $\mathrm{Ti}$ in the depletion region causing 
the oxygen ions to move out from the surface and increasing the dipole moment, an effect that would be strongest for monolayer films. Increased workfunctions and charge transfer have been predicted for the $\mathrm{TiO}_{2} / \mathrm{Mo}(100)$ interface which would be expected to be similar [19]. Second and subsequent layers would be in a more normal environment and would cause relaxation and reduction of the total dipole moment allowing the workfunction to converge down to its bulk value.

\section{Vibrational spectroscopy}

Figure 7 shows a typical FT-IR spectra of a 33ML thick film with two sharp bands at $448 \mathrm{~cm}^{-1}$ and $368 \mathrm{~cm}^{-1}$ and an intense broad band at $829 \mathrm{~cm}^{-1}(45.6 \mathrm{meV}$, $55.6 \mathrm{meV}$ and $102.8 \mathrm{meV}$ ). The positions of the three bands are in good agreement with high resolution electron energy loss spectroscopy (HREELS) work on $\mathrm{TiO}_{2}(110)$ surfaces in which Fuchs-Kliewer surface phonon modes were found at 46, 54 and $95 \mathrm{meV}$ with $\sim 7 \mathrm{meV}$ resolution [42, 43]. As HREELS is a charged particle spectroscopy the samples have to be made slightly reduced to prevent significant charging leading to some slight changes to the electronic structure. At low incident beam energies these phonon modes were seen to shift by $2 \mathrm{meV}$ to lower energy in non-stoichiometric surfaces [43]. Egdell and co workers further investigated this effect by electron stimulated desorption of surface oxygen and also found that modes shifted to lower energy as the surface region became more defective[44]. The positions of the sharp low energy phonon modes do not vary with film thickness (in the 6-35ML range covered) even though the XPS indicates that thinner films appear slightly less stoichiometric. The breadth of the mode at $829 \mathrm{meV}$ precludes accurate identification of any shifts. The lack of a shift in position with apparent stoichiometry further indicates that the charge transfer responsible for the apparent non- 
stoichiometry is located at the tungsten-titania interface and does not play a role in the bulk or surface properties.

Figure 8 shows the relative strength of the modes as a function of film thickness. The increasing strength with film thickness is expected as the surface phonon modes extend well into the bulk and thus sample the whole of the ultra-films. Figure 9 shows the temperature dependence of the phonon frequencies for the two sharpest lines. Temperatures below $273 \mathrm{~K}$ were externally calibrated from the recorded output from the C-type thermocouple and in-head transmitter used in the experiment and a K-type thermocouple attached to the sample in air and are hence subject to significant uncertainties. The lowering of the frequencies could be due to mismatched thermal expansion of the substrate and overlayer or be an intrinsic property of $\mathrm{TiO}_{2}$ due to lattice expansion. The thermal expansion coefficients of rutile and tungsten differ by about $50 \%\left(7.25\right.$ and $4.5 \times 10^{-6}$ respectively). Also plotted are data from reference [45] which are from infra-red reflectivity measurements for single crystal $\mathrm{TiO}_{2}$. The coefficients of expansion would tend to increase the relative compression of the rutile lattice with temperature leading to phonon mode frequencies consistently above those of a single crystal. The close match of the temperature dependencies of the phonon frequencies indicate that the film behaves as a relaxed single crystal and any departure from bulk behaviour is negligible.

\section{Conclusions}

We have shown that the growth of ultrathin $\mathrm{TiO}_{2}$ films on $\mathrm{W}(100)$ surfaces results in the epitaxial growth of a rutile structure which has been extensively characterised by photoemission and vibrational spectroscopies. Upon oxide film growth the tungsten surface states are removed and the thinnest films appear non- 
stoichiometric in XPS. However, in UPS measurements no new bandgap derived features at $\sim 0.9 \mathrm{eV}$ binding energy, which would be characteristic of occupied $3 \mathrm{~d}$ defect states, are resolved. This paradox is resolved by consideration of the band bending at the interface. The workfunction of the tungsten increases by $\sim 1.3 \mathrm{eV}$ for the thinnest films which is in accord with theoretical predictions for a charge transfer from surface to the film and also gives rise to the $\mathrm{Ti}^{3+}$ states observed in XPS. The UPS data shows that the valence band onset was consistently found $2.85 \mathrm{eV}$ below the Fermi level for thicker films but band bending due to the charge transfer pushes these states to higher binding energy in the thinner films. The vibrational structure of the overlayer was investigated by high resolution synchrotron far infra-red spectroscopy and the phonon bands recorded appeared indistinguishable from that expected of a purely rutile stoichiometric single crystal. The buried metal approach to vibrational spectroscopy was successful in this system and it appears to make an ideal model which would allow for high resolution vibrational spectroscopy on adsorbed molecules on the oxide surface.

\section{Acknowledgements}

The authors would like to thank the CCLRC for beamtime at the Daresbury Synchrotron, the Royal Society/Wolfson Foundation for infrastructure and the University of Reading for a studentship for JSM. MAN would like to thank the University of Reading for a visiting scientist position and the ESRF for time to pursue this work.

\section{References}

${ }^{1}$ U. Diebold, Surf. Sci. Rep. 48, 53, (2003). 
${ }^{2}$ L. A. Bursill and B. G. Hyde, in Progress in Solid State Chemistry, ed. H. Reiss and J. O. McCaldin 7, p. 177 (Pergamon Press, New York, 1972).

${ }^{3}$ G.S. Rohrer, V.E. Henrich and D.A. Bonnell, Science 250, 1239 (1990).

${ }^{4}$ G.S. Rohrer, V.E. Henrich and D.A. Bonnell, Surf. Sci. 278, 146 (1992).

${ }^{5}$ R.A. Bennett, S. Poulston, P. Stone and M. Bowker, Phys. Rev. B 59, 15, 1034110346 (1999).

${ }^{6}$ H. Nörenberg, R.E. Tanner, K.D. Schierbaum, S. Fischer and G.A.D. Briggs, Surf. Sci. 396, 52 (1998).

${ }^{7}$ H. Nörenberg and G.A.D. Briggs, Surf. Sci. 402-404, 738-741 (1998).

${ }^{8}$ S. Altieri, L. H. Tjeng, and G. A. Sawatzky, Phys. Rev. B 61, 16948 (2000).

${ }^{9}$ G. Pacchioni, L. Giordano, and M. Baistrocchi, Phys. Rev. Lett. 94, 226104 (2005).

${ }^{10}$ T. Schroeder, J.B. Giorgi, M. Bäumer and H.-J. Freund, Phys. Rev. B 66, 165422 (2002).

${ }^{11}$ N. D. McCavish and R. A. Bennett, Surf. Sci. 546, 47-56 (2003).

12 D. Guo, Q. Guo, M.S. Altman and E.G. Wang, J. Phys. Chem. B 109, 20968 (2005).

${ }^{13}$ T.V. Ashworth and G. Thornton, Thin Solid Films, 400, 43 (2001).

${ }^{14}$ T. V. Ashworth, C. A. Muryn and G. Thornton, Nanotechnology, 16, 3041 (2005).

${ }^{15}$ F. Sedona, G.A. Rizzi, S. Agnoli, F.X. Llabrés i Xamena, A. Papageorgiou, D. Ostermann, M. Sambi, P. Finetti, K. Schierbaum and G. Granozzi, J. Phys. Chem. B 109, 24411 (2005).

${ }^{16}$ F. Sedona, S. Agnoli and G. Granozzi, J. Phys. Chem. B 110, 15359 (2006).

${ }^{17}$ C.T. Campbell, Phys. Rev. Lett., 96, (2006) 066106.

${ }^{18}$ B. Montanari and N.M. Harrison, J. Phys. Condens. Matter 16, 273 (2004).

${ }^{19}$ L. Giordano, F. Cinquini and G. Pacchioni, Phys. Rev. B 73, 045414 (2005). 
${ }^{20}$ R.A. Bennett and N.D. McCavish, Top. in Catal., 36, 11-19 (2005).

${ }^{21}$ D.A. Slater, P. Hollins, M.A. Chesters, J. Pritchard, D.H. Martin, M. Surman, D.A. Shaw and I.H. Munro, Rev. Sci. Instrum. 63, 1547 (1992).

${ }^{22}$ M. Surman, J. Flaherty, I. Burrows, T.S Nunney, A.J. Roberts, A.J. Carew, K. Middleman, R. Raval, N.E. Wilson and A.E. Russell, Accelerator Based Sources of Infrared and Spectroscopic Applications, G. Lawrence Carr and P. Dumas, Editors, Proc. SPIE, 3775, 156 (1999).

${ }^{23}$ http://www.casaxps.com/

${ }^{24}$ M. P. Seah and W. A. Dench, Surf. and Interf. Anal. 1, 2 (1979).

25 N. Moslemzadeh, S.D. Barrett, V.R. Dhanak and G. Miller, Vacuum 67, 429 (2002).

${ }^{26}$ W. C. Mackrodt, E. A. Simson, and N. M. Harrison, Surf. Sci. 384 (1997) 192.

${ }^{27}$ S. Krischok, O. Hofft, and V. Kempter, Surf. Sci. 507, 69 (2002).

${ }^{28}$ J. Muscat, N. M. Harrison, and G. Thornton, Phys. Rev. B 59, 15457 (1999).

${ }^{29}$ S. Munnix and M. Schmeits, Phys. Rev. B 31, 3369 (1985).

${ }^{30}$ C. D. Valentin, G. Pacchioni, and A. Selloni, Phys. Rev. Lett. 97, 166803 (2006).

${ }^{31}$ D. Vogtenhuber, R. Podloucky and A. Neckel, Phys. Rev. B 49, 2099 (1994).

${ }^{32}$ K. D. Schierbauma, S. Fischera, M. C. Torquemadab, J. L. de Segoviab, E. Románc and J. A. Martín-Gagoc, Surf. Sci. 345, 3 (1996).

${ }^{33}$ K. Onda, B. Li, J. Zhao, K. D. Jordan, J. Yang, H. Petek, Science 308, 1154 (2005).

${ }^{34}$ H. Petek, APS Meeting Abstracts, 35009 (2004).

${ }^{35}$ D. Ostermann, G. Walther, and K. D. Schierbaum, Phys. Rev. B 71, 235416 (2005).

${ }^{36}$ K. Onda, B. Li, and H. Petek, Phys. Rev. B 70 (2004).

${ }^{37}$ Y. W. Chung, W. J. Lo, and G. A. Somorjai, Surf. Sci. 64, 588 (1977). 
${ }^{38}$ S. M. Sze, Semiconductor Devices Physics and Technology (John Wiley \& Sons, New York, 1985).

${ }^{39}$ A. T. Paxton and L. Thien-Nga, Phys. Rev. B 57, 1579 (1998).

${ }^{40}$ T. Okazawa, M. Kohyama, and Y. Kido, Surf. Sci. 600, 4430 (2006).

${ }^{41}$ A. Michaelides, P. Hu, M. H. Lee, A. Alavi, and D. A. King, Phys. Rev. Lett. 90, 246103 (2003).

${ }^{42}$ L.L. Kesmodel and J.A. Gates, Phys. Rev. B 23, 489 (1981).

${ }^{43}$ G. Rocker, J.A. Schaefer and W. Göpel, Phys. Rev. B 30, 3704 (1984).

${ }^{44}$ S. Eriksen and R.G. Egdell, Surf. Sci. 180, 263 (1987).

${ }^{45}$ F. Gervais and B. Piriou, Phys. Rev B 10, 1642 (1974).

Table I. Values and constraints used to fit the $\mathrm{Ti}^{4+}$ and $\mathrm{Ti}^{3+}$ spin orbit split states for the Ti 2p XPS spectra.

\begin{tabular}{ccccc}
\hline \hline Component & State & Position (eV) & Area & FWHM \\
$\mathrm{A}$ & $\mathrm{Ti}^{4+} 2 \mathrm{p}_{3 / 2}$ & 458.95 & Unconstrained & Unconstrained \\
$\mathrm{B}$ & $\mathrm{Ti}^{4+} 2 \mathrm{p}_{1 / 2}$ & $\mathrm{~A}+5.54$ & $\mathrm{~A}^{*} 0.5$ & Unconstrained \\
$\mathrm{C}$ & $\mathrm{Ti}^{3+} 2 \mathrm{p}_{3 / 2}$ & $\mathrm{~A}-1.9$ & Unconstrained & $\mathrm{A}^{*} 1$ \\
$\mathrm{D}$ & $\mathrm{Ti}^{3+} 2 \mathrm{p}_{1 / 2}$ & $\mathrm{~A}+3.54$ & $\mathrm{C}^{*} 0.5$ & $\mathrm{~B}^{*} 1$ \\
\hline
\end{tabular}



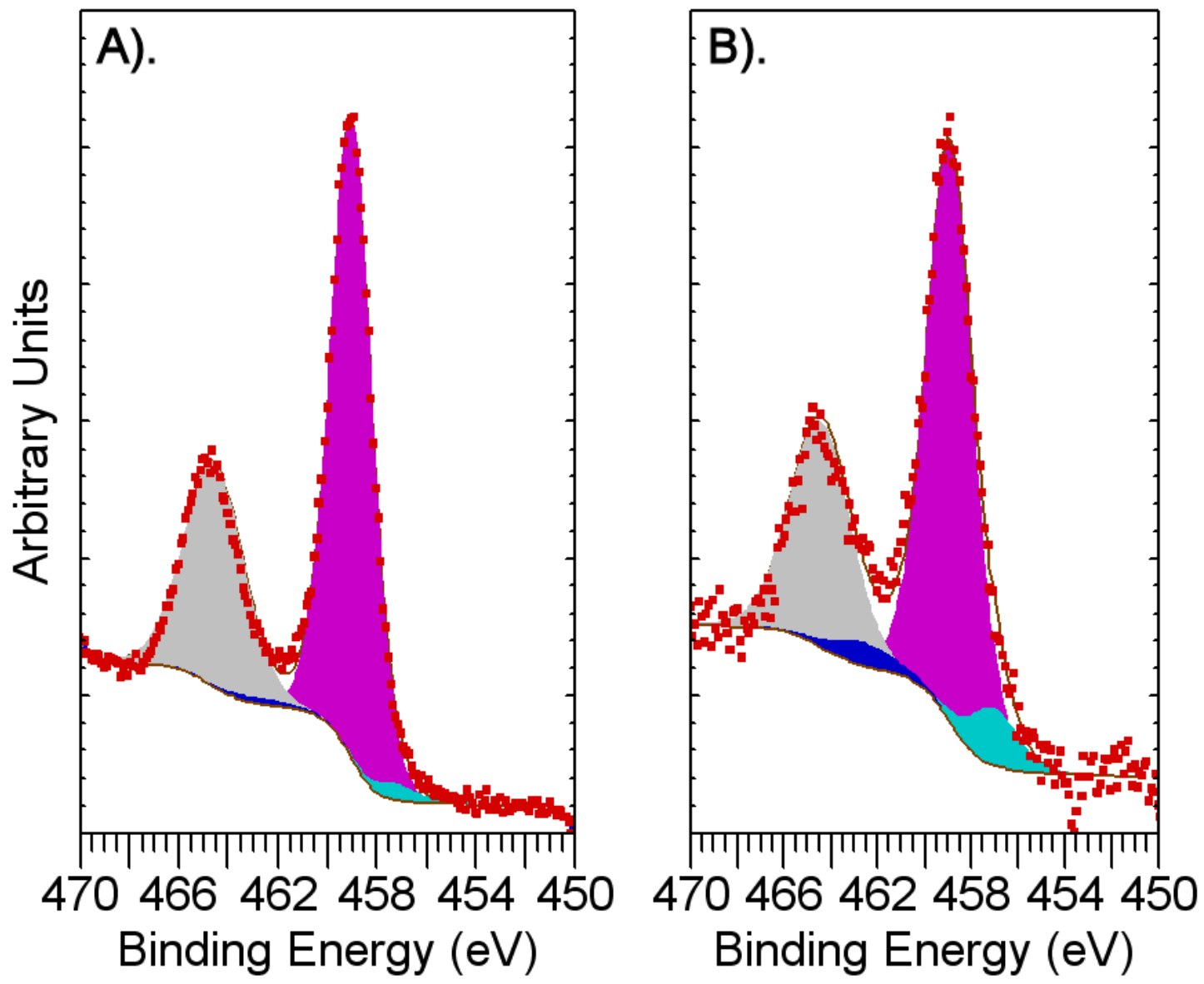

Figure 1. XPS spectra of the $\mathrm{Ti} 2 \mathrm{p}$ region for $\mathrm{A}) .30 \mathrm{ML} \mathrm{TiO}_{2}$ on $\mathrm{W}(100)$ and $\left.\mathrm{B}\right)$. 1ML $\mathrm{TiO}_{2}$ on $\mathrm{W}(100)$ with components fitted to quantify the $\mathrm{Ti}^{4+}$ and $\mathrm{Ti}^{3+}$ states (and their spin orbit split equivalents). For films $<10 \mathrm{ML}$ the XPS spectra show a marked increase in $\mathrm{Ti}^{3+}$ states in the shoulder at 457 (and $463 \mathrm{eV}$ ). 


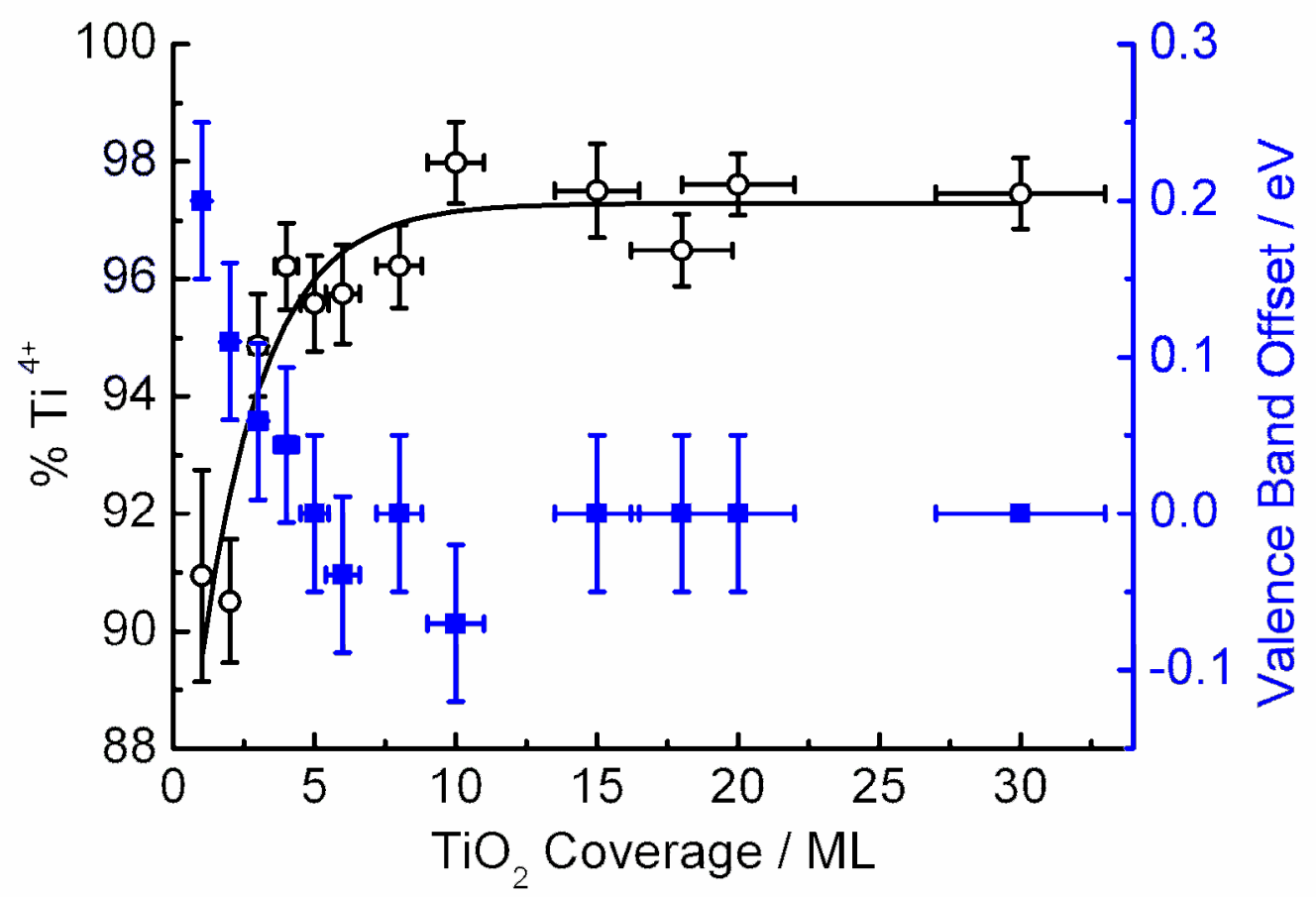

Figure 2. Plot showing the fitted fraction of $\mathrm{Ti}^{4+}$ ions (circles) and the valence band offsets (squares) present in the $\mathrm{TiO}_{2}$ films of thicknesses $1-30 \mathrm{ML}$. The stoichiometry are quoted as percentages of the relative proportion of $\mathrm{Ti}^{4+}$ in the sample and are fitted with a first order exponential curve (uncertainties in the stoichiometry were calculated through Monte Carlo simulations and are $10 \%$ in film thickness). Valence band offsets calculated through direct comparison to the pseudo-bulk and are $>0 \mathrm{eV}$ for films $<4 \mathrm{ML}$. For films $\geq 5 \mathrm{ML}$ no discernable offset is observed and valence emission begins at $2.85 \pm 0.05 \mathrm{eV}$. Uncertainties in the valence band emission are equal to the resolution observed at the Fermi edge and are estimated at $10 \%$ for the film thickness. 

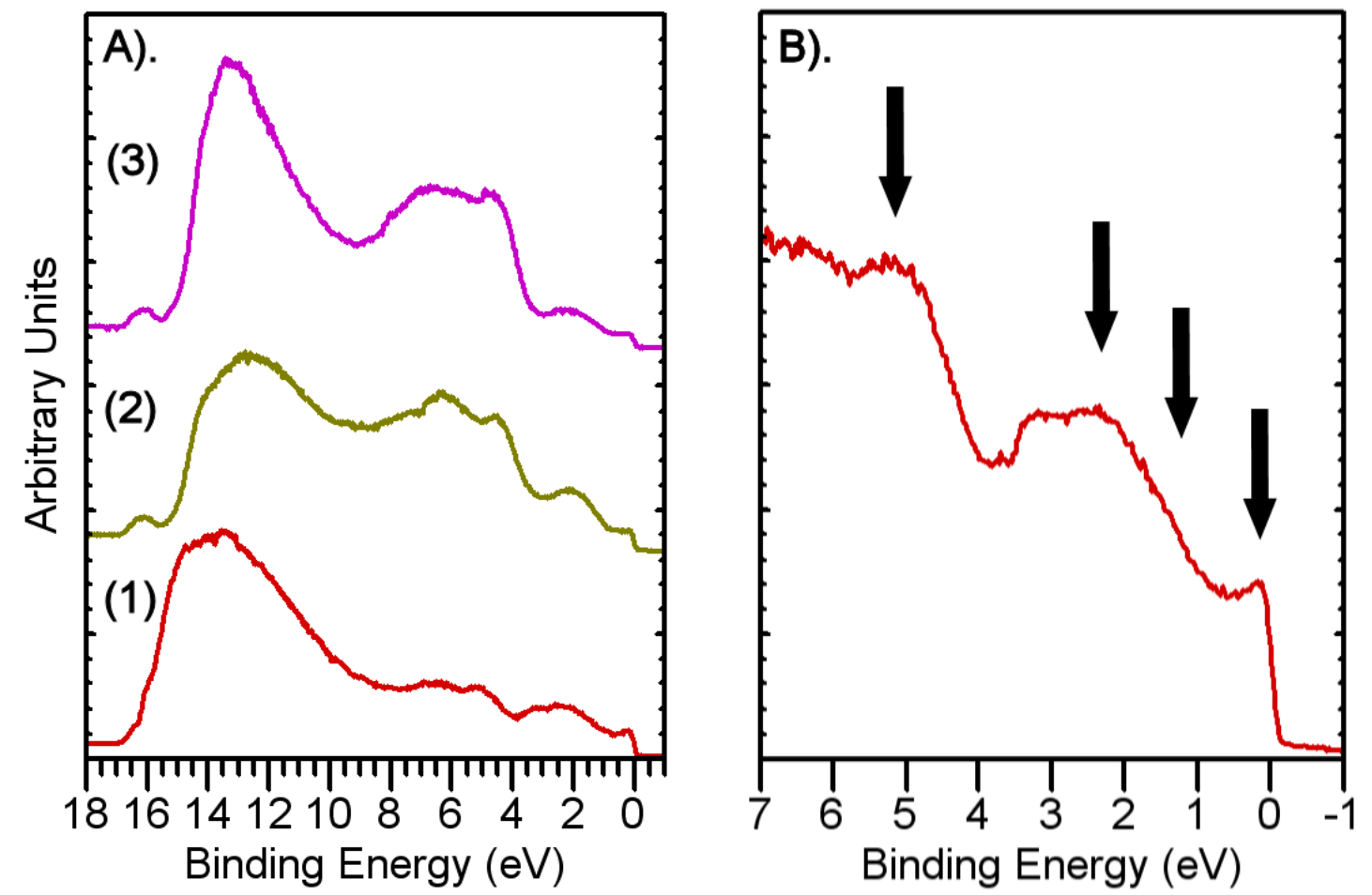

Figure 3. A). UPS spectra of (1) clean $\mathrm{W}(100)$, (2) $\mathrm{O}-\mathrm{W}(2 \times 1)$ reconstruction and (3) $1 \mathrm{ML} \mathrm{TiO}_{2}$ on $\mathrm{O}-\mathrm{W}(2 \times 1)$. B). Enlarged UPS spectra of clean W(100) showing the energies of $\mathbf{4}$ characteristic surface states. 


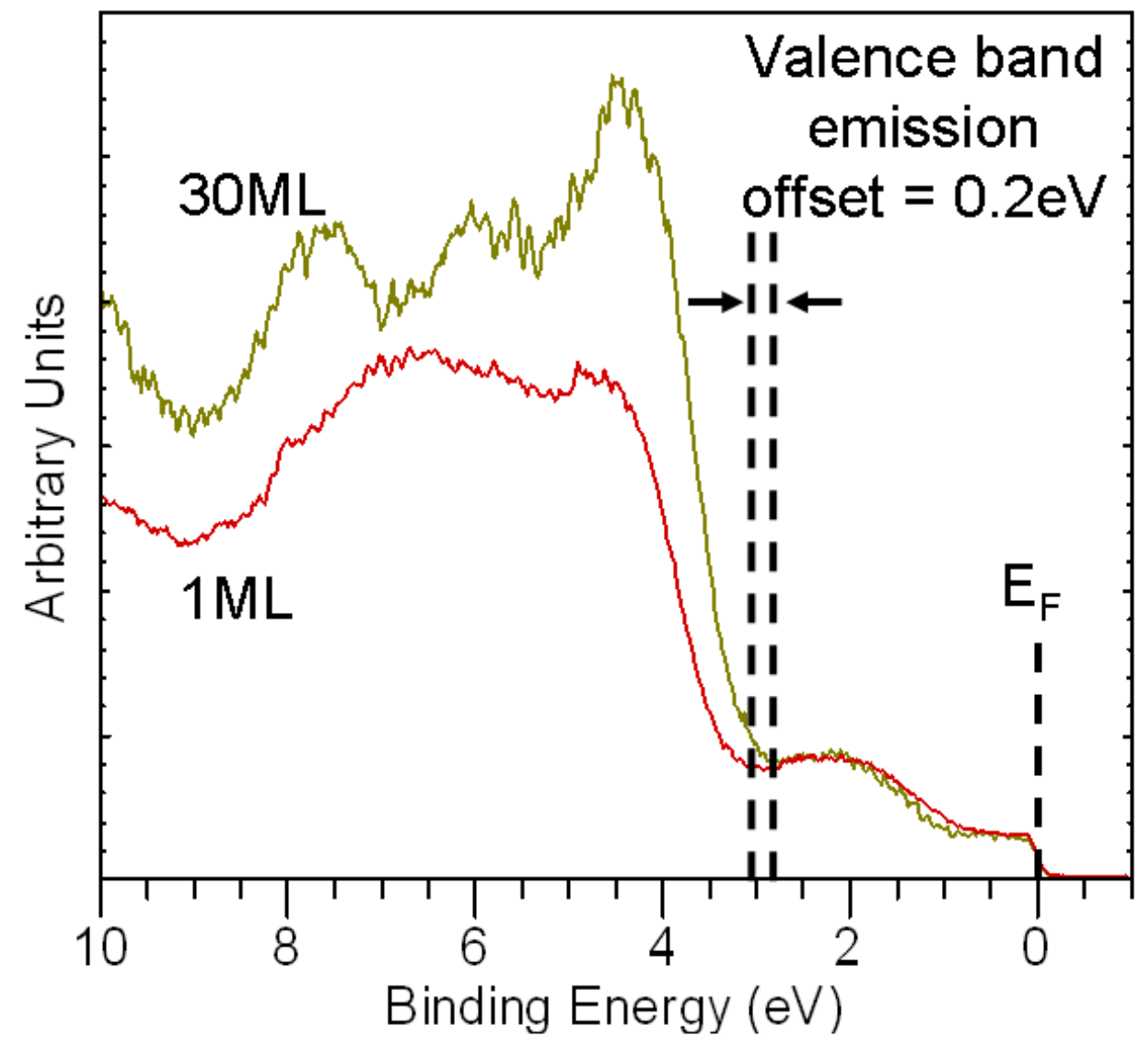

Figure 4. Overlaid $\mathrm{He}$ I UPS spectra for $1 \mathrm{ML}$ and $30 \mathrm{ML} \mathrm{TiO}_{2}$ films on W (100) normalised at the Fermi level displaying the development of structure within the valence band emission and the shift to lower binding energies with increasing film thickness. 


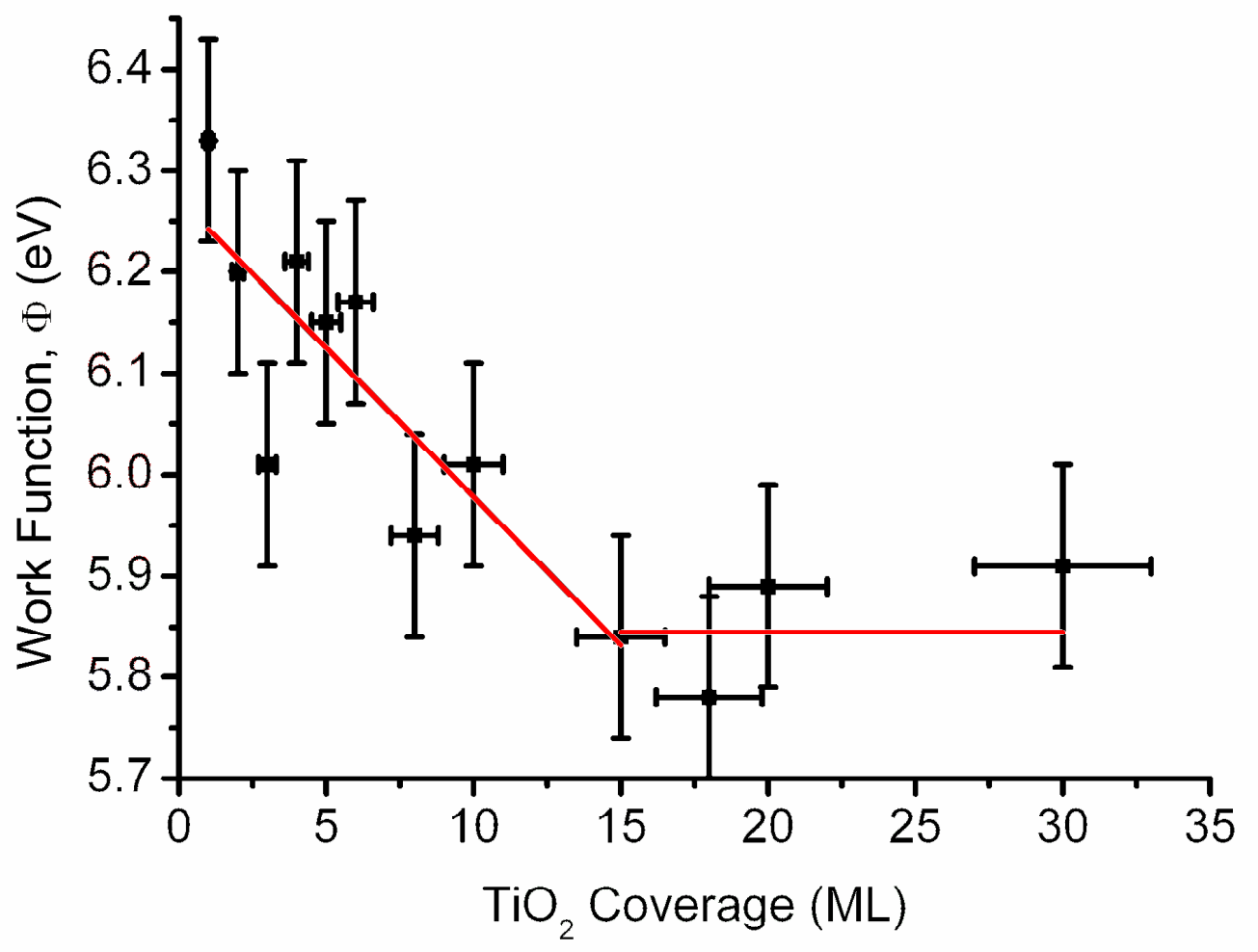

Figure 5. Work function values as a function of thickness for $\mathrm{TiO}_{2}$ films on W(100) showing an increase of $\sim 0.2-0.5 \mathrm{eV}$ for films $<10 \mathrm{ML}$ and a trend toward a constant value of $\sim 5.8 \mathrm{eV}$ for films $>15 \mathrm{ML}$. 


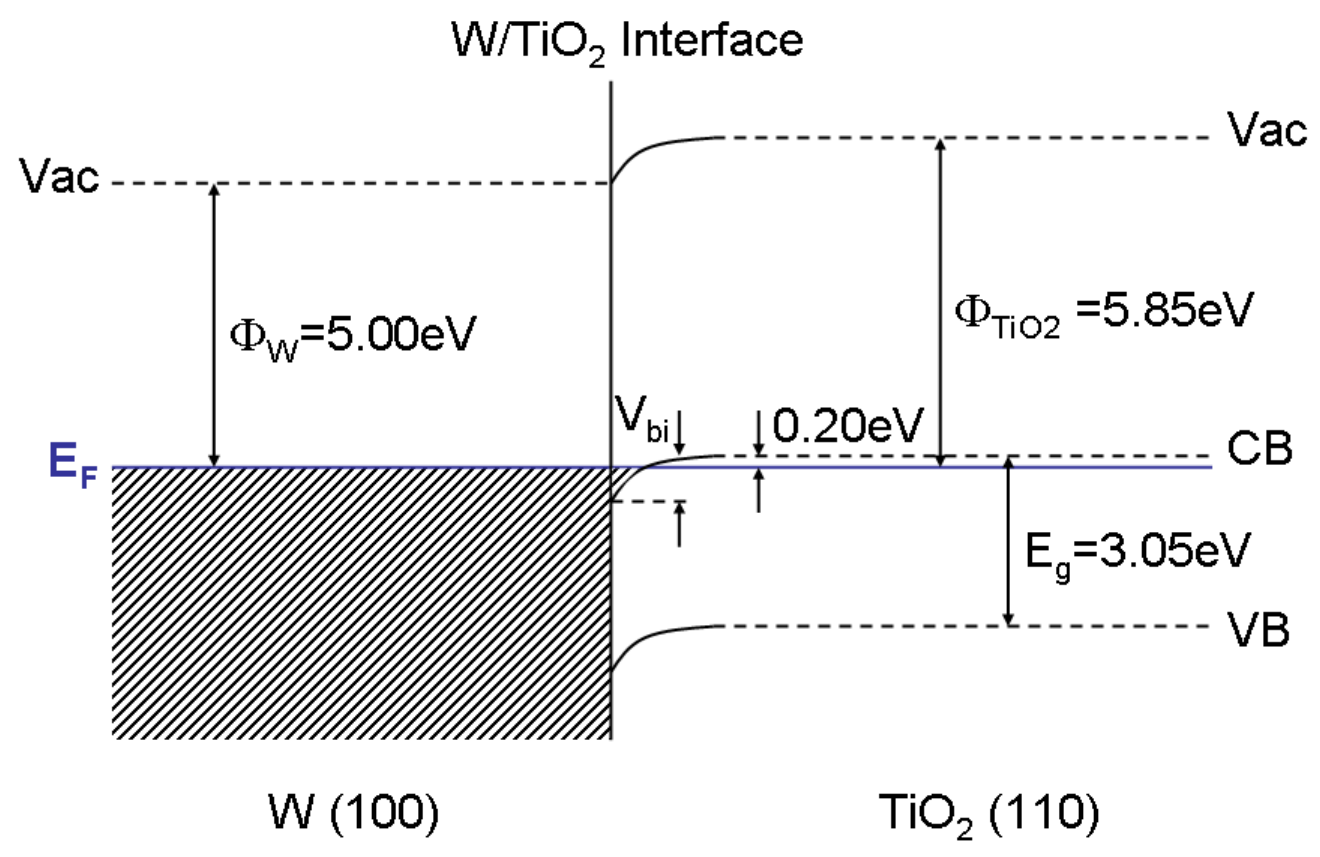

Figure 6. Band bending at the $\mathrm{W} / \mathrm{TiO}_{2}$ interface showing downward band bending and the crossing of the $\mathrm{TiO}_{2}$ conduction band minima with the Fermi level creating an accumulation layer and interface states. 


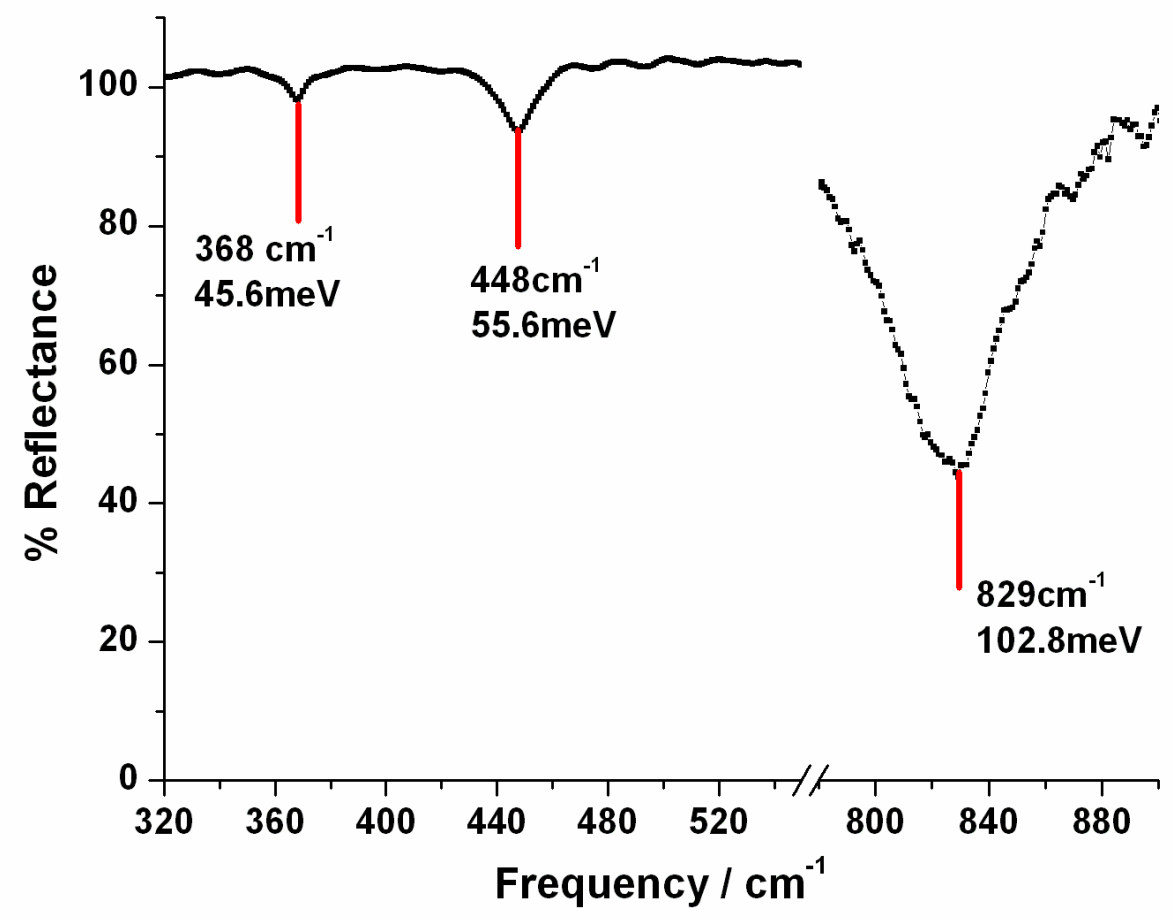

Figure 7. FT-IR spectra of a 33ML film at room temperature showing three Fuchs-Kliewer phonon modes. 


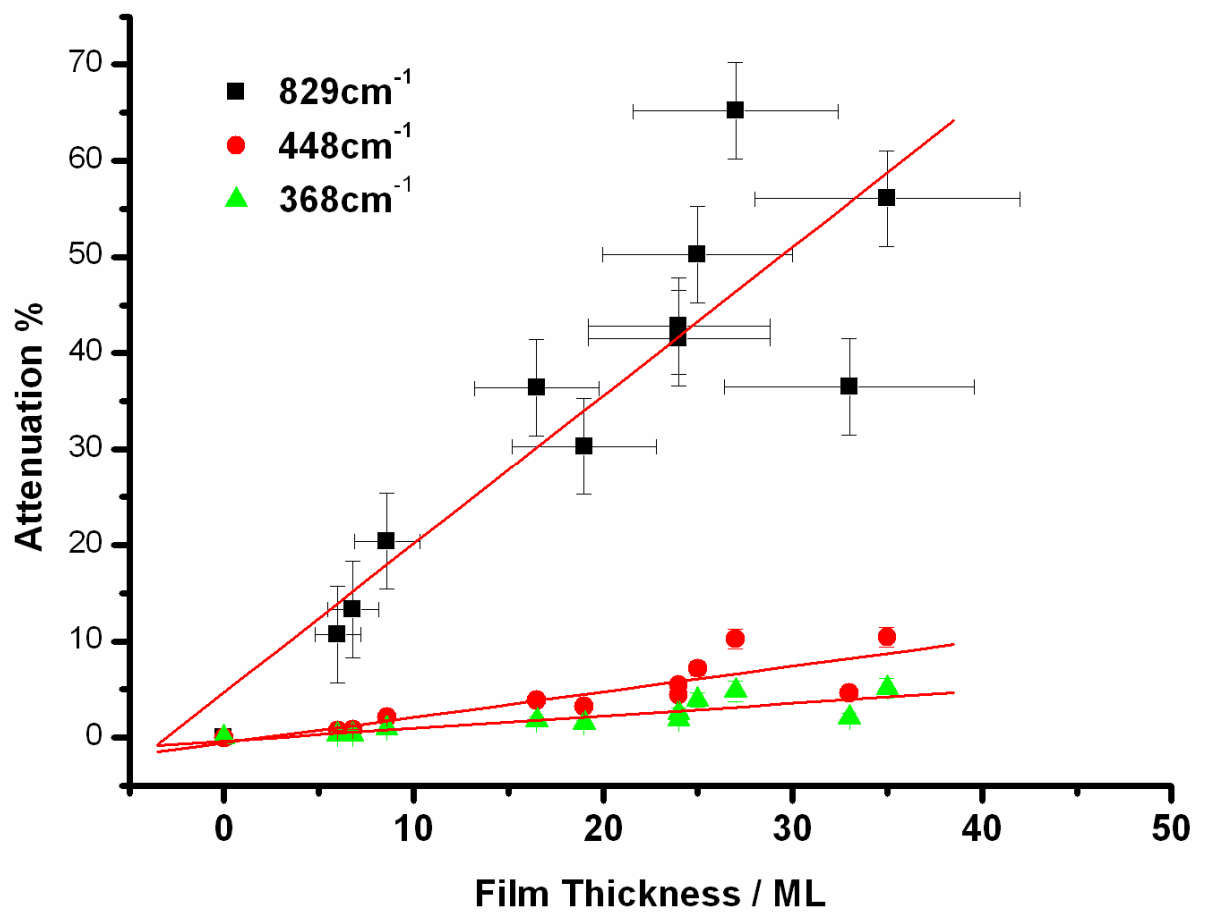

Figure 8. The relative strength of the three phonon modes as functions of film thickness. The straight lines are best linear fits as guides to the eye showing. Conservative error bars are given for uncertainty in film thickness and noise levels. 


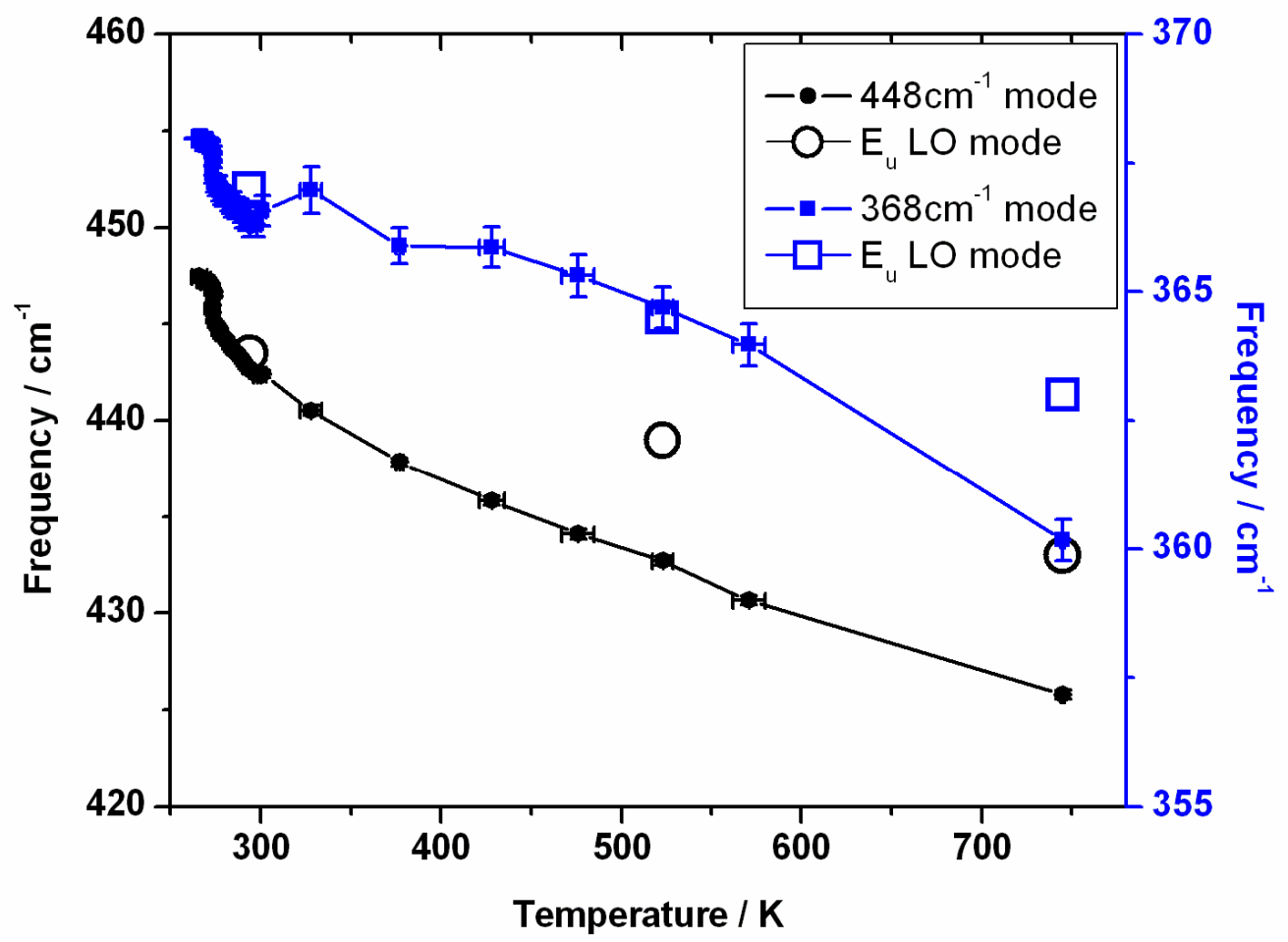

Figure 9 shows the temperature dependence of the phonon frequencies for the two sharpest modes of a 22ML film. In open symbols the values plotted are taken from IR reflectivity data from reference [45]. 